\title{
Eficacia de tres tipos de surfactante exógeno en prematuros con enfermedad de membrana hialina
}

\author{
LUIS ALFONSO MENDOZA T. ${ }^{1}$, MÓNICA OLIVEROS G. ${ }^{2}$, MIGUEL ÁNGEL OSORIO R. ${ }^{3}$, \\ MARTHA ARIAS G. ${ }^{4}$, YOLIMA RUÍZ S. ${ }^{2}$, DIANA ARCE C. ${ }^{2}$, DIANA ALZATE ${ }^{2}$ \\ 1. Pediatra Neonatólogo. Fundación Hospital San José de Buga. Unidad Central del Valle, Tuluá, Colombia. \\ 2. Terapeuta Respiratorio. Fundación Hospital San José de Buga, Colombia. \\ 3. Pediatra Neonatólogo. Universidad del Valle, Colombia. \\ 4. Pediatra. Fundación Hospital San José de Buga, Colombia.
}

\section{ABSTRACT \\ Effectiveness of three types of exogenous surfactant in preterm infants with hyaline membrane disease}

Introduction: Hyaline membrane disease is an important cause of neonatal mortality. The objective of this research is to evaluate the efficacy of three different exogenous surfactants in premature infants. Patients and Method: A retrospective cohort analysis in 93 preterm infants $\geq 24$ weeks and birth weight $\geq 500 \mathrm{~g}$ was performed, 31 infants for each surfactant. Exposure consisted of the 1st dose of bovactant (Alveofact ${ }^{\circledR}$ ) $50 \mathrm{mg} / \mathrm{kg}$, beractant $\left(\right.$ Survanta $\left.^{\circledR}\right) 100 \mathrm{mg} / \mathrm{kg}$ initially, and poractant alfa $\left(\right.$ Curosurf $\left.^{\circledR}\right) 200 \mathrm{mg} / \mathrm{kg}$. The variables included duration of mechanical ventilation, duration of oxygen therapy, hospital stay, need for second dose of surfactant, adverse events surfactant administration and prematurity complications. Mortality and bronchopulmonary dysplasia (BPD) were evaluated. Statistical analysis was performed using Stata ${ }^{\circledR} 11.0, \chi^{2}$ or Fisher exact test for qualitative variables and ANOVA or Kruskal-Wallis tests for quantitative and association relative risk, all with $95 \%$ confidence level. Results: There were no gender, weight and gestational age differences at birth among the three groups. No statistically significant differences were found regarding duration of mechanical ventilation, duration of oxygen therapy, administration of a second dose of surfactant, hospital stay and complications among the three groups. Adverse events related to surfactant administration occurred for beractant and poractant alpha. There were $30(32.3 \%)$ deaths, $8(25.8 \%)$ associated to bovactant, $10(32.3 \%)$ to beractant and $12(38.7 \%)$ to poractant alpha ( $\mathrm{p}>0.05)$. Mortality and/or BDP occurred in $10(32.2 \%)$ infants who received bovactant, $10(32.2 \%)$ beractant and $14(45.2 \%)$ with poractant alpha $(\mathrm{p}>0.05)$. Conclusions: The primary and secondary outcomes among the three surfactants tested were similar, taking into account the limitations of the work.

(Key words: Exogenous surfactant, preterm, hyaline membrane disease, complications, bronchopulmonary dysplasia).

Rev Chil Pediatr 2013; 84 (6): 616-627

Recibido el 15 de agosto de 2012, devuelto para corregir el 10 de diciembre de 2012, segunda versión 12 de febrero de 2013 , tercera versión 18 de marzo de 2013, aceptado para publicación el 23 de septiembre de 2013.

Este trabajo cumple con los requisitos sobre consentimiento /asentimiento informado, comité de ética, financiamiento, estudios animales y sobre la ausencia de conflictos de intereses según corresponda.

Correspondencia a:

Luis Alfonso Mendoza Tascón

E-mail: lamendozat@gmail.com 


\section{RESUMEN}

Introducción: La enfermedad de membrana hialina es causa importante de mortalidad neonatal. El objetivo de esta investigación fue evaluar la eficacia de tres tipos de surfactante exógeno en prematuros. Pacientes y Método: Estudio de cohorte retrospectiva, en 93 neonatos prematuros, $\geq 24$ semanas $\mathrm{y} \geq 500 \mathrm{~g}$ de peso al nacer, 31 para cada surfactante. La exposición fue la administración de $1^{a}$ dosis bovactant (Alveofactß) 50 $\mathrm{mg} / \mathrm{kg}$, beractant (Survanta $\left.{ }^{\circledR}\right) 100 \mathrm{mg} / \mathrm{kg}$ inicial, y poractant alfa (Curosurf®) $200 \mathrm{mg} / \mathrm{kg}$. Las variables en estudio incluyeron tiempo de ventilación mecánica, tiempo de oxigenoterapia, estancia hospitalaria, necesidad de segunda dosis de surfactante, eventos adversos por la administración del surfactante y complicaciones por prematuridad. Además, se evaluó mortalidad, displasia broncopulmonar (DBP) y mortalidad o DBP. Análisis estadístico mediante Stata ${ }^{\circledR} 11.0$, empleando $\chi^{2}$ o Prueba Exacta de Fisher para variables cualitativas y Pruebas ANOVA o Kruskal-Wallis para cuantitativas y riesgo relativo para las asociaciones, todas con su intervalo de confianza de $95 \%$. Resultados: No hubo diferencias para sexo, peso y edad gestacional al nacer entre los 3 grupos. No se hallaron diferencias estadísticamente significativas para tiempo de ventilación mecánica, tiempo de oxigenoterapia, administración de una segunda dosis de surfactante, estancia hospitalaria y complicaciones entre los 3 grupos. Los eventos adversos por administración de surfactante se presentaron para beractant y poractant alfa. Ocurrieron $30(32,3 \%)$ muertes, $8(25,8 \%)$ para bovactant, $10(32,3 \%)$ beractant y $12(38,7 \%)$ poractant alfa $(\mathrm{p}>0,05)$. La mortalidad y/o DBP ocurrió en $10(32,2 \%)$ neonatos con bovactant, $10(32,2 \%)$ con beractant y $14(45,2 \%)$ con poractant alfa $(p>0,05)$. Conclusiones: Los resultados primarios y secundarios entre los tres surfactantes evaluados fueron muy similares, teniendo en cuenta las limitaciones del trabajo. (Palabras clave: Surfactante exógeno, prematuro, enfermedad de membrana hialina, complicaciones, displasia broncopulmonar).

Rev Chil Pediatr 2013; 84 (6): 616-627

\section{Introducción}

La enfermedad de membrana hialina (EMH), es una de las complicaciones que causa mayor mortalidad en prematuros ${ }^{1}$, siendo la administración de surfactante exógeno la terapia estándar en esta patología. Son múltiples los estudios que establecen los beneficios de este tratamiento en la mejoría de la sobrevida, displasia broncopulmonar y reducción de la incidencia del neumotórax ${ }^{2-5}$. Son varios los surfactantes comerciales existentes, siendo los más usados el Poractant alfa (Curosurf®) y el beractant (Survanta ${ }^{\circledR}$ ), y otros menos usados en nuestro país como el bovactant (Alveofact $\left.{ }^{\circledR}\right)$. La mayoría de los estudios se han realizado con beractant y Poractant alfa, los cuales se han comparado a diferentes dosis ${ }^{6}$, mientras la mayoría de trabajos con bovactant, son observacionales y realizados en Europa.

El objetivo de este trabajo fue comparar la eficacia en términos de tiempo de ventilación, tiempo de oxigenación, eventos adversos y complicaciones, de la administración de tres tipos de surfactante, Poractant alfa, beractant y bovactant, a las dosis recomendadas actualmente y estandarizadas en la Unidad de Cuidados Intensivos Neonatales de la Fundación Hospital San José de Buga, Colombia, entre los años 2005 y 2012.

\section{Pacientes y Método}

\section{Diseño}

Se efectuó un análisis de los datos de prematuros que recibieron surfactante entre el 19 de septiembre de 2005 y 28 de febrero de 2012. Se incluyeron neonatos de ambos sexos con peso al nacer entre 500 y 1.999 g, edad gestacional entre 23 y 35 semanas, quienes por peso $(<1.000 \mathrm{~g})$ o por diagnóstico de EMH (dificultad respiratoria y radiografía tórax con infiltrados bilaterales en vidrio esmerilado, broncograma aéreo y pérdida de volumen pulmonar) se les suministró surfactante profiláctico o terapéutico, respectivamente. Se excluyeron neonatos con neumonía, sepsis, asfixia, trisomías y malformaciones congénitas mayores. 


\section{Pacientes}

La muestra estuvo constituida por todos los neonatos que cumplieran con los criterios de inclusión y no tuviesen al menos uno de exclusión, y que fue extraída de una base de datos con 182 neonatos de todos los pesos y edades gestacionales que recibieron surfactante, tanto por enfermedad de membrana hialina, como por otras causas. De estos, se excluyeron 57 pacientes por tener más de $1.999 \mathrm{~g}$ de peso al nacer. Entre los neonatos elegibles, se excluyeron 25 neonatos con neumonía y sepsis, 5 con asfixia perinatal y un neonato con hernia diafragmática y uno por dismorfismo. En total, se incluyeron 93 neonatos, 31 para cada tipo de surfactante.

La recolección de la información que se encontró en la base de datos, se realizó mediante interrogatorio de la madre, evaluación del neonato y los datos de laboratorio o radiología directamente de los informes. Las historias clínicas materna y neonatal fueron una fuente secundaria de información. Este estudio fue aprobado por el Comité de Ética de la institución.

\section{Surfactante}

El método de administración del surfactante fue bolo rápido en infusión por tubo orotraqueal o por sonda orotraqueal. Las dosis y métodos de administración se realizaron de acuerdo las recomendaciones de los manufacturadores y las diferentes publicaciones o consensos, bovactant o Alveofact $\AA$ (SF-R1, Boehringer In-gelheim Ltd, Germany) $(1,2 \mathrm{ml} /$ $\mathrm{kg} /$ dosis; $50 \mathrm{mg} / \mathrm{kg} /$ dosis total de fosfolípido, para la primera y las dosis adicionales), beractant o Survanta ${ }^{\circledR}$ (Ross-Abbott Laboratories, Ohio, USA) (4 ml/kg/dosis; $100 \mathrm{mg} / \mathrm{kg} /$ dosis total de fosfolípidos, para la primera y las dosis adicionales) y Poractant alfa o Curosurf® (Chiesi farmaceutici, Italy) $(2,5 \mathrm{ml} / \mathrm{kg} / \mathrm{dosis}$; $200 \mathrm{mg} / \mathrm{kg} /$ dosis total de fosfolípido para la primera dosis y $100 \mathrm{mg} / \mathrm{kg}$ dosis total de fosfolípido para la segunda dosis).

\section{Terapia ventilatoria}

La terapia ventilatoria convencional fue empleada mediante modalidad asistida-controlada o sincrónica, de manera estandarizada, mientras el destete del ventilador se hizo cuando el neonato requiriera $\mathrm{FiO}_{2}<0,3$, frecuencia ventilatoria $\leq 20$ por min, presión ventilatoria inspiratoria pico $(\mathrm{PIP}) \leq 15 \mathrm{cmH}_{2} \mathrm{O}$, presión

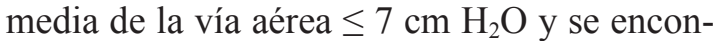
trara clínica y metabólicamente estable. Los criterios de egreso hospitalario incluyeron para los que vivían en zona urbana un peso de 1.800 g y zona rural o de difícil acceso a un centro de salud con $2.000 \mathrm{~g}$, que se alimentaran adecuadamente, que no tuvieran dependencia al oxígeno y que termorregularan adecuadamente.

Para cada neonato se registraron nombre, entidad de salud, número de historia clínica, fecha de nacimiento y de ingreso, peso y edad gestacional al nacer medido por fecha de última menstruación, ecografía obstétrica más temprana y Puntaje de Ballard, sexo, gestación múltiple, administración prenatal de surfactante, vía de nacimiento, valoración de APGAR a los 1, 5 y 10 min, diagnósticos, reanimación cardio-respiratoria, calificación del grado de dificultad respiratoria mediante la Escala de Silverman, causa de prematuridad, edad de aplicación de primera y segunda dosis de surfactante, tiempo entre última dosis de surfactante y extubación, PIP, presión al final de la espiración (PEEP), presión media de la vía aérea (Paw), fracción inspirada de oxígeno $\left(\mathrm{FIO}_{2}\right)$, presión arterial de oxígeno $\left(\mathrm{PaO}_{2}\right)$, Índice de Oxigenación $\left(\mathrm{FiO}_{2}\right.$ x Paw x100/ $\left.\mathrm{PaO}_{2}\right)$, Índice de Kirby $\left(\mathrm{PaO}_{2} / \mathrm{FiO}_{2}\right)$ al momento de la administración del surfactante, a las 2 y $6 \mathrm{~h}$ después de administrado el surfactante, modo ventilatorio, tipo de surfactante administrado, tiempo de suministro de oxígeno, dosis de surfactante en centímetros cúbicos y $\mathrm{mg} / \mathrm{kg}$ de peso y total, aplicación prenatal de betametasona y número de dosis, así como el tiempo entre la última dosis de betametasona y el nacimiento, complicaciones por prematuridad y efectos adversos por la administración del surfactante y mortalidad.

\section{Variables en estudio}

Los resultados primarios incluyeron tiempo de ventilación mecánica y tiempo de suministro de oxígeno. Los resultados secundarios fueron necesidad de segunda dosis de surfactante, terapia postnatal con esteroides 
para tratamiento de displasia broncopulmonar, estancia hospitalaria, efectos adversos inmediatos durante la administración del surfactante (desaturación, taquicardia, bradicardia, hipotensión o hipertensión arterial), complicaciones como retinopatía de la prematuridad, hemorragia intraventricular severa grado III o IV, sepsis, enterocolitis necrotizante estados $\geq$ IIA, ductus arterioso permeable que requiera terapia farmacológica o quirúrgica, hemorragia pulmonar, síndromes de escape en los primeros 14 días (presencia de neumotórax u otro síndrome de escape en tórax o pulmones). Adicionalmente, se evaluó la incidencia de displasia broncopulmonar (DBP) y mortalidad entre los tres tipos de surfactantes.

\section{Análisis estadístico}

Para la descripción de las variables categóricas se emplearon las frecuencias absolutas y relativas, mientras para las cuantitativas el promedio y su desviación estándar o la mediana y el rango intercuartil, de acuerdo a su distribución. La significancia estadística de las diferencias entre los surfactantes Bovactant, Beractant y Poractant alfa fue realizado para las variables categóricas o nominales mediante pruebas de $\chi^{2}$ o Exacto de Fisher y para las variables cuantitativas mediante pruebas ANOVA o Kruskall-Wallis. Los efectos de los tres surfactantes sobre los resultados se realizaron calculando el Riesgo Relativo (RR) con su intervalo de confianza de 95\%. Los análisis estadísticos se hicieron mediante el programa estadístico Stata ${ }^{\circledR}$ 11.0. La significancia estadística fue definida como un valor $\mathrm{p}<0,05$.

\section{Resultados}

\section{Características generales}

Un total de 93 neonatos fueron incluidos en el estudio. Los varones fueron $57,5 \%$, sin diferencia entre los grupos. La mediana de peso al nacer fue $1.300 \mathrm{~g}$ (RI: $970-1.670 \mathrm{~g}$ ) y a pesar de que en el grupo de beractant fueron de mayor peso, no se halló diferencia estadísticamente significativa. La mediana de la edad gestacional fue 30 semanas (rango intercuartil [RI]: 27-32 semanas), siendo los de beractant
2 semanas mayores, sin diferencias para los tres grupos. Tampoco se halló diferencias para calificación de Apgar a 1, 5 y 10 min y administración de betametasona antenatal, aunque las madres de los neonatos que recibieron bovactant en menor proporción recibieron betametasona. Las causas principales de nacimiento prematuros fueron parto prematuro (31\%) y ruptura prematura de membranas ovulares $(24,1 \%)$ (tabla 1$)$.

\section{Soporte ventilatorio y tiempo de suministro de oxigenoterapia}

En cuanto al Índice de Kirby a las 2 y 6 $\mathrm{h}$ de administrada la primera dosis de surfactante, encontramos resultados muy similares para los tres surfactantes, con una ventaja para el beractant a las $2 \mathrm{~h}$ y para el Poractant alfa a las 6 h, sin embargo, no se logró demostrar diferencia estadísticamente significativa. Para el Índice de Oxigenación, tampoco logramos demostrar diferencias significativas entre los tres surfactantes. Encontramos que el Índice de Oxigenación a las $2 \mathrm{~h}$ de suministrado el surfactante para el bovactant tuvo un valor ligeramente superior $10,7 \pm 9,1$ respecto a los otros dos surfactantes, mientras que a las $6 \mathrm{~h}$ para el beractant este valor fue de $13,9 \pm 8,9$ valor mayor al de bovactant y Poractant alfa (tabla 2). El 75\% de los neonatos que tuvieron un Índice de Oxigenación menor a 10 habían recibido una segunda dosis de surfactante, ocurriendo esto en el $55,5 \%$ de los neonatos que recibieron bovactant, $50 \%$ de beractant y $90 \%$ de Poractant alfa (tabla 2).

El tiempo de ventilación mecánica fue para el bovactant 2,2 veces mayor que para el beractant y 2,4 veces mayor que para el Poractant alfa, diferencia estadísticamente significativa $(\mathrm{p}<0,05)$. Mientras tanto, el tiempo de oxigenoterapia fue 1,6 veces mayor para neonatos tratados con bovactant y berantant, comparado con el tiempo de oxigenoterapia de los neonatos que recibieron Poractant alfa (tabla 2).

\section{Administración de segunda dosis de surfactante}

El 40,1\% de los prematuros requirieron una segunda dosis del medicamento, sin hallar diferencias estadísticamente significativas entre 
Tabla 1. Características neonatales

\begin{tabular}{|c|c|c|c|c|}
\hline & $\begin{array}{c}\text { Bovactant } \\
1^{\text {a }} \text { dosis } 50 \mathrm{mg} / \mathrm{kg} \\
2^{\mathrm{a}} \text { dosis } 50 \mathrm{mg} / \mathrm{kg} \\
(\mathrm{n}=31)\end{array}$ & $\begin{array}{c}\text { Beractant } \\
\text { 1 }^{\text {a }} \text { dosis } 100 \mathrm{mg} / \mathrm{kg} \\
2^{\text {a }} \text { dosis } 100 \mathrm{mg} / \mathrm{kg} \\
(\mathrm{n}=31)\end{array}$ & 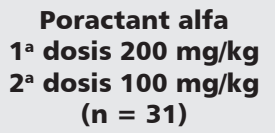 & $\mathbf{p}$ \\
\hline Hombres & $19(61,3 \%)$ & $17(60 \%)$ & $20(66,7 \%)$ & 0,730 \\
\hline $\begin{array}{l}\text { Peso al nacer (g) } \\
\text { Mediana (RI) }\end{array}$ & $\begin{array}{c}1.130 \\
(980-1.860)\end{array}$ & $\begin{array}{c}1.448 \\
(1.030-1.710)\end{array}$ & $\begin{array}{c}1.160 \\
(910-1.560)\end{array}$ & 0,418 \\
\hline $\begin{array}{l}\text { Edad gestacional al nacer } \\
\text { Mediana (RI) }\end{array}$ & $\begin{array}{c}29 \\
(26-32)\end{array}$ & $\begin{array}{c}31 \\
(28-32)\end{array}$ & $\begin{array}{c}29 \\
(27-32)\end{array}$ & 0,280 \\
\hline Apgar 1 min < 7 (RI) & $8(6-9)$ & $8(7-9)$ & $8(7-8)$ & 0,314 \\
\hline Apgar 5 min < 7 (RI) & $8(7-9)$ & $9(8-10)$ & $9(8-10)$ & 0,118 \\
\hline Apgar $10 \min <7$ (RI) & $9(8-10)$ & $10(9-10)$ & $9(8-10)$ & 0,225 \\
\hline Esteroides antenatales (12 óo 24 mg) & $9(29 \%)$ & $17(54,8 \%)$ & $16(51,6 \%)$ & 0,084 \\
\hline \multicolumn{5}{|l|}{ Causas de la prematuridad: } \\
\hline Trabajo de parto prematuro & $13(46,4 \%)$ & $8(25,8 \%)$ & $9(31 \%)$ & 0,356 \\
\hline Ruptura prematura de membranas & $0^{*}$ & $3(3,7 \%)$ & $7(24,1 \%)^{*}$ & 0,016 \\
\hline Preeclampsia & $5(17,9 \%)$ & $8(29,6 \%)$ & $4(13,8 \%)$ & 0,392 \\
\hline Infección materna & $4(14,3 \%)$ & $3(11,1 \%)$ & $5(17,2 \%)$ & 0,750 \\
\hline Otras causas & $5(17,9 \%)$ & $5(18,5 \%)$ & $4(13,8 \%)$ & 0,919 \\
\hline Sin datos & 4 & 7 & 2 & - \\
\hline
\end{tabular}

Rl: Rango intercuartil. * $p<0,05 ;$ g: gramos; min: minutos.

Tabla 2. Resultados primarios

\begin{tabular}{|c|c|c|c|c|}
\hline & $\begin{array}{c}\text { Bovactant } \\
1^{\text {a }} \text { dosis } 50 \mathrm{mg} / \mathrm{kg} \\
\text { 2 }^{\mathrm{a}} \text { dosis } 50 \mathrm{mg} / \mathrm{kg} \\
(\mathrm{n}=31)\end{array}$ & $\begin{array}{c}\text { Beractant } \\
\text { 1 }^{\text {a }} \text { dosis } 100 \mathrm{mg} / \mathrm{kg} \\
\text { 2 }^{\mathrm{a}} \text { dosis } 100 \mathrm{mg} / \mathrm{kg} \\
(\mathrm{n}=31)\end{array}$ & 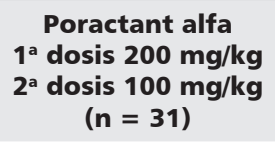 & $\mathbf{p}$ \\
\hline $\begin{array}{l}\text { Tiempo de ventilación mecánica } \\
\text { Mediana (RI) }\end{array}$ & $63,1( \pm 68,7)$ & $28,5( \pm 29,3)$ & $26,7( \pm 26,1)$ & 0,004 \\
\hline $\begin{array}{l}\text { Tiempo total de oxígeno (h) } \\
\text { Mediana (RI) }\end{array}$ & $138(74-364)$ & $139(42-208)$ & $89(56-355)$ & 0,641 \\
\hline $\begin{array}{l}\text { Paw } 2 \text { h después de surfactante } \\
\text { Promedio (DE) }\end{array}$ & $8,4( \pm 2,6)$ & $7,2( \pm 1,6)$ & $7,1( \pm 1,4)$ & 0,065 \\
\hline $\begin{array}{l}\text { Paw } 6 \text { h después de surfactante } \\
\text { Promedio (DE) }\end{array}$ & $8,9( \pm 2,1)^{*}$ & $7,6( \pm 1,7)^{*}$ & $6,6( \pm 1,7)^{*}$ & $<0,001$ \\
\hline $\begin{array}{l}\text { Indice de Kirby }\left(\mathrm{PaO}_{2} / \mathrm{FiO}_{2}\right) \text { inicial } \\
\text { Promedio (DE) }\end{array}$ & $180( \pm 66)$ & $168( \pm 85)$ & $164( \pm 81)$ & 0,775 \\
\hline $\begin{array}{l}\text { Indice de Kirby }\left(\mathrm{PaO}_{2} / \mathrm{FiO}_{2}\right) \text { a las } 2 \mathrm{~h} \\
\text { Promedio (DE) }\end{array}$ & $169( \pm 139)$ & $261( \pm 134)$ & $194( \pm 168)$ & 0,164 \\
\hline $\begin{array}{l}\text { Indice de Kirby }\left(\mathrm{PaO}_{2} / \mathrm{FiO}_{2}\right) \text { a las } 6 \mathrm{~h} \\
\text { Promedio (DE) }\end{array}$ & $123( \pm 119)$ & $125( \pm 117)$ & $223( \pm 148)$ & 0,164 \\
\hline $\begin{array}{l}\text { Indice de oxigenación }\left(\mathrm{IO}=\mathrm{FiO}_{2} \times\right. \\
\left.\text { Paw } \times 100 / \mathrm{PaO}_{2}\right) \text { inicial Promedio (DE) }\end{array}$ & $6,9( \pm 3,6)$ & $6,9( \pm 5,8)$ & $6,3( \pm 4,2)$ & 0,881 \\
\hline $\begin{array}{l}\text { Indice de oxigenación }\left(\mathrm{IO}=\mathrm{FiO}_{2} \mathrm{x}\right. \\
\left.\text { Paw } \mathrm{x} 100 / \mathrm{PaO}_{2}\right) \text { a las } 2 \mathrm{~h} \text { Promedio (DE) }\end{array}$ & $10,7( \pm 9,1)$ & $6,5( \pm 9)$ & $8,1( \pm 8,4)$ & 0,889 \\
\hline $\begin{array}{l}\text { Indice de oxigenación }\left(\mathrm{IO}=\mathrm{FiO}_{2} \mathrm{x}\right. \\
\left.\mathrm{Paw} \times 100 / \mathrm{PaO}_{2}\right) \text { a las } 6 \mathrm{~h} \text { Promedio (DE) }\end{array}$ & $8,2( \pm 7,7)$ & $13,9( \pm 8,9)$ & $6,1( \pm 6,4)$ & 0,127 \\
\hline
\end{tabular}

Rl: Rango intercuartíl. Paw: Presión media de la vía aérea. $\mathrm{PaO}_{2}$ : Presión arterial de oxígeno. $\mathrm{FiO}_{2}$ : Fracción inspirada de oxígeno. IO: Índice de oxigenación. DE: Desviación estándar. * $p<0,001$. 
los tres surfactantes. Los neonatos con peso $<1.000 \mathrm{~g}$ tuvieron mayor riesgo de recibir segunda dosis de surfactante (RR: 1,7; IC 95\%: $1,1-2,7 ; \mathrm{p}<0,05)$, al igual que los neonatos de $<1.500 \mathrm{~g}$ al nacer (RR: 2,3; IC 95\%: 1,4-4,3; $\mathrm{p}<0,05)$ (tabla 3). Por otro lado, hallamos una asociación estadísticamente significativa entre una segunda dosis y reducción de mortalidad neonatal (RR: 0,74; IC 95\%: 0,54-1,01; $\mathrm{p}=$ 0,047).

\section{Estancia hospitalaria}

La mediana de la estancia para todos los neonatos fue de 21 días (RI: 7-36 días), sin hallar diferencias para los tres surfactantes. Entre los sobrevivientes, una estancia superior a 21 días ocurrió en $9(39,1 \%), 6(27,3 \%)$ y 6 $(33,3 \%)$ de los neonatos que recibieron bovactant, beractant y Poractant alfa, respectivamente (tabla 3).

Administración profiláctica, de rescate, dosis total y costos de la administración del surfactante. Veinticinco (26,9\%) de los neonatos recibieron surfactante profilácticamente entre el momento de nacimiento y los primeros 30 min de vida, sin hallar diferencias entre los tres tipos de surfactantes. La dosis total de surfactante administrado fue significativamente mayor para el poractat alfa (mediana $190 \mathrm{mg} / \mathrm{kg}$;
RI: $186-200 \mathrm{mg} / \mathrm{kg}$ ), seguido por el beractant (mediana $122 \mathrm{mg} / \mathrm{kg}$; RI: 100-200 mg/kg), mientras que el de menor dosis fue bovactant (mediana $70 \mathrm{mg} / \mathrm{kg}$; RI: 50-100 mg/kg), siendo esta diferencia estadísticamente significativa entre bovactant y Poractant alfa $(p<0,05)$. De ahí que el costo del Poractant alfa resultara 2 y 2,6 veces mayor cuando se comparó con el beractant y bovactant, respectivamente (tabla 3 ).

\section{Eventos adversos y complicaciones asociadas a la administración del surfactante y a la prematuridad}

En cuanto a los eventos adversos durante la administración de surfactante como reducción de la saturación de oxígeno, bradicardia, hipotensión arterial y taquicardia, ocurrieron 32 casos $(34,4 \%)$. No hubo casos para el bovactant, mientras ocurrieron $4(12,9 \%)$ casos para beractant y $4(12,9 \%)$ casos para Poractant alfa (tabla 4).

Hubo 35 (37,6\%) complicaciones, siendo la principal la sepsis con 21 casos $(60 \%)$, neumotórax 4 casos $(4,3 \%)$, ductus arterioso permeable $3(3,2 \%)$, hemorragia intraventricular $2(2,1 \%)$ y enterocolitis estado clínico $\geq$ IIA 2 $(2,1 \%)$, sin diferencias estadísticamente significativas entre los tres surfactantes $(\mathrm{p}>0,05)$ (tabla 4).

Tabla 3. Resultados secundarios: tipo de administración de surfactante, dosis, estancia hospitalaria y costos del tratamiento

\begin{tabular}{|c|c|c|c|c|}
\hline & $\begin{array}{c}\text { Bovactant } \\
1^{\text {a }} \text { dosis } 50 \mathrm{mg} / \mathrm{kg} \\
2^{\mathrm{a}} \text { dosis } 50 \mathrm{mg} / \mathrm{kg} \\
(\mathrm{n}=31)\end{array}$ & $\begin{array}{c}\text { Beractant } \\
1^{\text {a }} \text { dosis } 100 \mathrm{mg} / \mathrm{kg} \\
2^{\mathrm{a}} \text { dosis } 100 \mathrm{mg} / \mathrm{kg} \\
(\mathrm{n}=31)\end{array}$ & $\begin{array}{c}\text { Poractant alfa } \\
\text { 1 }^{\text {a }} \text { dosis } 200 \mathrm{mg} / \mathrm{kg} \\
2^{\text {a }} \text { dosis } 100 \mathrm{mg} / \mathrm{kg} \\
(\mathrm{n}=31)\end{array}$ & $\mathbf{p}$ \\
\hline Surfactante profiláctico, n (\%) & $8(25,8 \%)$ & $7(22,6 \%)$ & $10(32,2 \%)$ & 0,684 \\
\hline Surfactante de rescate, $\mathrm{n}(\%)$ & $23(74,2 \%)$ & $24(77,4 \%)$ & $21(67,8 \%)$ & 0,684 \\
\hline $\begin{array}{l}\text { Edad primera dosis (min) } \\
\text { Mediana (RI) }\end{array}$ & $45(10-180)$ & $67(15-300)$ & $35(15-120)$ & 0,421 \\
\hline Segunda dosis, n (\%) & $12(38,7 \%)$ & $12(38,7 \%)$ & $15(48,4 \%)$ & 0,673 \\
\hline $\begin{array}{l}\text { Estancia hospitalaria en días } \\
\text { Mediana (RI) }\end{array}$ & $21(7-45)$ & $22(13-31)$ & $18(4-36)$ & 0,789 \\
\hline $\begin{array}{l}\text { Total dosis de surfactante } \mathrm{mg} / \mathrm{kg} \\
\text { Mediana (RI) }\end{array}$ & $70(50-110)^{*}$ & $122(100-200)$ & $190(186-300)^{*}$ & 0,000 \\
\hline $\begin{array}{l}\text { Costo del tratamiento en US } \$ \\
\text { con surfactante (RI) }\end{array}$ & $\begin{array}{c}414,8^{* *} \\
(296,3-651,8)\end{array}$ & $\begin{array}{c}535^{* *} \\
(428-856)\end{array}$ & $\begin{array}{c}1.067,2^{* *} \\
(1.044,8-1.685,1)\end{array}$ & $<0,05$ \\
\hline
\end{tabular}

RI: Rango intercuartíl. ${ }^{*} p<0,001 .{ }^{* *} p<0,05$. 
Tabla 4. Resultados secundarios: eventos adversos y complicaciones

\begin{tabular}{|c|c|c|c|c|}
\hline & 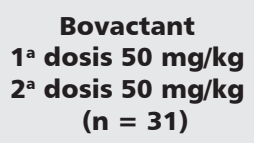 & $\begin{array}{c}\text { Beractant } \\
\text { 1 }^{\text {a }} \text { dosis } 100 \mathrm{mg} / \mathrm{kg} \\
\text { 2 }^{\mathrm{a}} \text { dosis } 100 \mathrm{mg} / \mathrm{kg} \\
(\mathrm{n}=31)\end{array}$ & 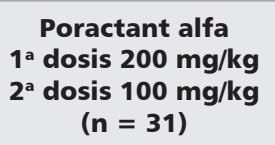 & $\mathbf{p}$ \\
\hline $\begin{array}{l}\text { Eventos adversos asociadas a la } \\
\text { administración del surfactante }\end{array}$ & 0 & $4(12,9 \%)$ & $4(12,9 \%)$ & 0,112 \\
\hline Bradicardia, n (\%) & 0 & $1(3,2 \%)$ & $3(9,7 \%)$ & 0,161 \\
\hline Desaturación, n (\%) & 0 & $2(6,4 \%)$ & $2(6,4 \%)$ & 0,352 \\
\hline Hipotensión arterial, n (\%) & 0 & $1(3,2 \%)$ & 0 & 0,364 \\
\hline Taquicardia, n (\%) & 0 & 0 & 0 & - \\
\hline $\begin{array}{l}\text { Complicaciones asociados a la } \\
\text { prematuridad, } \mathrm{n}(\%)\end{array}$ & $12(38,7 \%)$ & $7(22,6 \%)^{*}$ & $17(54,8 \%)^{*}$ & 0,031 \\
\hline Neumotórax, n (\%) & $2(6,4 \%)$ & 0 & $2(6,4 \%)$ & 0,352 \\
\hline Ductus arteriosos permeable, n (\%) & $2(6,4 \%)$ & 0 & $1(3,2 \%)$ & 0,356 \\
\hline Hemorragía intraventricular III o IV, n (\%) & 0 & 0 & $2(6,4 \%)$ & 0,130 \\
\hline Enterocolitis $\geq \| \mathrm{A}, \mathrm{n}(\%)$ & 0 & $1(3,2 \%)$ & $1(3,2 \%)$ & 0,560 \\
\hline Sepsis, n (\%) & $6(19,4 \%)$ & $6(19,4 \%)$ & $9(29 \%)$ & 0,550 \\
\hline
\end{tabular}

Tabla 5. Mortalidad y displasia broncopulmonar

\begin{tabular}{|c|c|c|c|c|}
\hline & 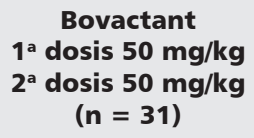 & 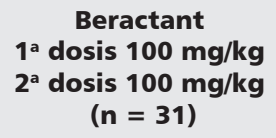 & 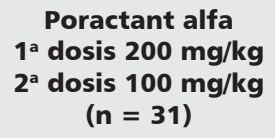 & $\mathbf{p}$ \\
\hline Mortalidad, n (\%) & $8(25,8 \%)$ & $10(32,3 \%)$ & $12(38,7 \%)$ & 0,503 \\
\hline $\begin{array}{l}\text { Mortalidad primeras } 48 \mathrm{~h} \text { de vida, } \\
\mathrm{n}(\%)\end{array}$ & $1(3,2 \%)$ & $4(12,9 \%)$ & $5(16,1 \%)$ & 0,233 \\
\hline $\begin{array}{l}\text { Displasia broncopulmonar } \\
\text { a los } 28 \text { días de vida, n (\%) }\end{array}$ & $2(6,4 \%)$ & 0 & $2(6,4 \%)$ & 0,352 \\
\hline Mortalidad y DBP, n (\%) & $10(32,2 \%)$ & $10(32,2 \%)$ & $14(45,2 \%)$ & 0,476 \\
\hline
\end{tabular}

DBP: Displasia broncopulmonar.

\section{Mortalidad y DBP}

La mortalidad ocurrió en $30(32,3 \%)$ neonatos, presentándose $12(38,7 \%), 10(32,3 \%)$ y $8(25,8 \%)$ muertes para tratados con Poractant alfa, beractant y bovactant, respectivamente. En las primeras $48 \mathrm{~h}$ de vida, para bovactant ocurrió $1(3,2 \%)$ muerte, para beractant 4 $(12,9 \%)$ y para Poractant alfa $5(16,1 \%)$, sin demostrar diferencia estadísticamente significativa. Se identificaron $4(4,3 \%)$ casos de DBP, ocurriendo $2(6,4 \%)$ casos entre los neonatos que recibieron bovactant y Poractant alfa y ninguno para beractant. Respeto al resultado mortalidad y/o DBP no se halló diferencia entre los tres grupos (tabla 5).

\section{Discusión}

Este trabajo tuvo por objetivo evaluar la eficacia y comparar los resultados neonatales de la administración de tres tipos de surfactantes, a tres dosis diferentes, que son las recomendadas por los manofacturadores, la Academia 
Americana de Pediatría y los Lineamientos del Consenso Europeo de Manejo de Enfermedad de Membrana Hialina en Prematuros de 2010 y empleadas en diferentes trabajos de investigación ${ }^{6,7}$, bajo la hipótesis de que no habían diferencias estadísticamente significativas entre los tres surfactantes. Las características de nuestros pacientes son similares a la informada por otros trabajos ${ }^{6,8,9}$. Sin embargo, hubo un menor número de madres que recibieron betametasona antenatal en el grupo de bovactant, mientras los neonatos del grupo de beractant fueron de mayor peso, dado que en nuestra unidad de recién nacidos aplicamos los otros dos surfactantes a los neonatos más pequeños, bajo la suposición que por el volumen del beractant pueden presentar más complicaciones durante la administración.

Respecto a la ventilación mecánica y la oxigenoterapia, hallamos que para el bovactant las h de ventilación mecánica se prolongaron más de dos veces y la presión media de la vía aérea a las 6 h después de administrado el surfactante fue significativamente mayor que para los otros dos surfactantes exógenos, esto quizás esté asociado a la menor dosis administrada de este. No se logró demostrar diferencias para los otros parámetros de ventilación y de oxigenación. Otros autores, demostraron diferencias significativas en la presión media de la vía aérea favoreciendo al poractant alfa al compararlo con beractant ${ }^{10,11}$, la cual fue menor entre las primeras $24-72 \mathrm{~h}$. Singh N y cols. ${ }^{6}$, informan en su meta-análisis una disminución de los requerimientos de $\mathrm{O}_{2}$ para el poractant alfa a $200 \mathrm{mg} / \mathrm{kg}$ cuando se le comparó con beractant $100 \mathrm{mg} / \mathrm{kg}$. Proquitté $\mathrm{H}$ y cols. ${ }^{12}$, al comparar el tratamiento en prematuros $<32$ semanas con $100 \mathrm{mg} / \mathrm{kg}$ de bovactant y poractant alfa, no hallaron diferencias estadísticamente significativas para tiempo de oxigenoterapia, tiempo de ventilación mecánica, así como tampoco para número de dosis administradas, dosis acumulada, complicaciones (síndromes de escape aéreo, hemorragia pulmonar, leucomalacia periventricular, hemorragia intraventricular grado III o IV, ductus arteriosos permeable, DBP), estancia hospitalaria. Baroutis G y cols. ${ }^{8}$, tampoco hallaron diferencias estadísticamente significativas entre bova- ctant, poractant alfa y beractant a dosis de 100 $\mathrm{mg} / \mathrm{kg}$ para oxigenoterapia $\left(\mathrm{FiO}_{2}\right.$ y duración), presión media de la vía aérea y días de ventilación mecánica. Estos hallazgos son similares a los informados por Yalaz M y cols. ${ }^{9}$, quienes compararon bovactant con beractant.

En cuanto a la redosificación de surfactante, el $40,1 \%$ de los neonatos requirieron una dosis adicional, siendo los neonatos $<1.500 \mathrm{~g}$ el grupo que requirió más de una dosis. Nuestro trabajo encontró que una segunda dosis está asociada con una reducción de la mortalidad neonatal. El surfactante puede comenzar rápidamente a ser metabolizado y la inactivación funcional puede ocurrir, resultado de la acción de proteínas solubles y otros pequeños factores de la vía aérea pequeña y del alveolo ${ }^{13-16}$.

Los conceptos patogénicos de los factores pre y postnatales y los mecanismos inflamatorios en la enfermedad pulmonar neonatal se han discutido recientemente ${ }^{17}$. La corioamnionitis se ha identificado como uno de los principales factores asociados al trabajo de parto prematuro. Más de $50 \%$ de los partos prematuros inmaduros se ha expuesto a corioamnionitis, microorganismos y productos microbianos en el útero, así como a la expresión aumentada de citoquinas proinflamatorias, con la presencia de factores de interferencia del aclaramiento de líquido pulmonar, ocurriendo un mayor proceso de apoptosis de células de la vía aérea, con severa injuria pulmonar aguda y deterioro de la respuesta al surfactante debido a la inactivación y disfunción ${ }^{17}$. Esto ha llevado a que varios informes promuevan el tratamiento con redosificación o dosis múltiples de surfactante $\mathrm{e}^{18,19}$, favoreciendo la reducción de neumotórax (RR típico: 0,51; IC 95\%: 0,30-0,88) y disminuyendo la mortalidad (RR típico: 0,63; IC 95\%: 0,39-10,2 $)^{20}$. Un trabajo europeo informó que una segunda dosis de surfactante (incluyendo bovactant, poractant alfa y beractant) fue necesaria en el $43 \%$ de los neonatos a la mediana de edad de $17 \mathrm{~h}$ (RI: 11-27 h). Los que recibieron poractant alfa, una segunda dosis fue necesaria en $42 \%$, beractant $41 \%$ y bovactant $63 \%{ }^{21}$. Estas cifras son superiores a las informadas en nuestro trabajo. Para Singh $\mathrm{N}$ y cols., la incidencia de redosificación fue significativamente más baja para el grupo de 
Poractant alfa a $200 \mathrm{mg} / \mathrm{kg}$ cuando se comparó con beractant a $100 \mathrm{mg} / \mathrm{kg}$ (RR: 0,71; IC 95\%: $0,57-0,88 ; p<0,05)$. En prematuros $<30$ semanas y $<1.250 \mathrm{~g}$, con enfermedad de membrana hialina, la estrategia de dosis múltiples con surfactante exógeno de origen animal, produjo mejores resultados respecto a oxigenación $\mathrm{y}$ requerimientos ventilatorios, con menor riesgo de neumotórax (RR típico 0,51; IC 95\%: 0,30 a 0,88 ; diferencia de riesgo típico: $-0,09$; IC 95\%: $-0,15$ a $-0,02)$ y una tendencia hacia una menor mortalidad (RR típico: 0,63; IC 95\%: 0,39 a 1,02; diferencia de riesgo típico: $-0,07$; IC del 95\% -0,14, 00,00) .

La estancia hospitalaria estuvo alrededor de tres semanas, mientras que el $22,6 \%$ permanecieron hospitalizados por sobre este tiempo, sin demostrar diferencia entre los tres tipos de surfactantes. Para Singh N y cols., la estancia hospitalaria fue significativamente más corta en los prematuros tratados con Poractant alfa a $200 \mathrm{mg} / \mathrm{kg}$ al compararlos con beractant a 100 $\mathrm{mg} / \mathrm{kg}$ (diferencia media ponderada: - 9,9; IC 95\%: - 29,96 a 9,16; $\mathrm{p}<0,001)^{6}$.

En nuestro trabajo, las indicaciones y los modos de administración del surfactante, son basadas en las recomendaciones publicadas en la literatura. No se halló diferencia en este aspecto entre los tres tratamientos. Los tiempos de administración de la primera dosis de surfactante mostraron que $25(26,9 \%)$ de los neonatos recibieron la primera dosis profilácticamente entre los 0-30 min de vida, mientras los restantes con una mediana de $180 \mathrm{~min}$ de edad (RI: 60-570 min) de vida, mientras para la segunda dosis tuvo una mediana de $7,5 \mathrm{~h}$ (RI: 6-12 h) de aplicación. La primera y segunda dosis de surfactante en cuanto al tiempo fue comparable a la descrita por otros trabajos. Koch L y cols., al comparar en neonatos de 23 a 26 semanas de gestación entre la administración de surfactante profiláctico (primeros 5 min de vida) y de rescate sólo hallaron diferencias estadísticamente significativa en el tiempo de ventilación mecánica $(74 \pm 26$ h vs $171 \pm 35$ $\mathrm{h} ; \mathrm{p}<0,05)^{23}$. Moura C y cols., al comparar la administración de surfactante entre $0-2 \mathrm{~h}$, más de 2 h hasta 6 h y más de $6 \mathrm{~h}$, hallaron que los neonatos que recibieron el surfactante en las primeras $2 \mathrm{~h}$ de vida tuvieron menos síndromes de escape aéreo, menor tiempo de oxigenoterapia y de ventilación mecánica $(\mathrm{p}<0,05)^{22}$.

Hallamos diferencias estadísticamente significativas en la dosis acumulada de bovactant, beractant y Poractant alfa. En este trabajo la mediana de la dosis total para bovactant fue $70 \mathrm{mg} / \mathrm{kg}$ (RI: $50-110 \mathrm{mg} / \mathrm{kg}$ ), en contraste de beractant $122 \mathrm{mg} / \mathrm{kg}$ (RI: $100-200 \mathrm{mg} / \mathrm{kg}$ ) y Poractant alfa $190 \mathrm{mg} / \mathrm{kg}$ (RI: $186-300 \mathrm{mg} /$ $\mathrm{kg}$ ) que fue consistentemente más elevado. Los volúmenes instilados en la primera dosis de surfactante para bovactant (promedio 1,9 \pm $0,8 \mathrm{ml}$ ), beractant (promedio $5,9 \pm 1,7 \mathrm{ml}$ ) y Poractant alfa (promedio 1,9 $\pm 0,5 \mathrm{ml}$ ), fueron diferentes debido a las diferentes concentraciones de los surfactantes (bovactant: $50 \mathrm{mg}$ en 1,2 ml, beractant: $25 \mathrm{mg}$ en $1 \mathrm{ml}$ y Poractant alfa $120 \mathrm{mg}$ en $1,5 \mathrm{ml}$ ). La mayor dosis recomendada actualmente de Poractant alfa $\mathrm{y}$ el costo del medicamento por mg, hicieron que este resultara más costoso que los otros dos surfactantes, diferencia que fue estadísticamente significativa.

Los eventos adversos observados durante la administración de los surfactantes se presentaron para pacientes con beractant $(12,9 \%)$ y Poractant alfa $(12,9 \%)$, tal vez asociado a los mayores volúmenes necesariamente instilados. Nuestros resultados son ligeramente superiores a los informados por Speer CP y cols., quienes describen problemas con la administración de beractant y no con Poractant alfa, probablemente reflejando el mayor volumen de dosis del beractant. El reflujo del beractant fue observado en $3 / 40(7,5 \%)$ y una disminución de la saturación por debajo de $85 \%$ en 2/40 (5\%) 5 min después de la administración del surfactante ${ }^{11}$. Se encontró mayor número de complicaciones neonatales como neumotórax, ductus arterioso permeable, hemorragia intraventricular III o IV, DBP, enterocolitis necrotizante estado IIA o mayor y sepsis, para los neonatos tratados con Poractant alfa, diferencia que no fue significativa al compararlo con el bovactant, pero si al compararlo con el beractant. El 54,8\% de los pacientes tratados con Poractant alfa, vs 38,7\% con bovactant y $22,6 \%$ con beractant presentaron alguna complicación clínica. Igualmente, los pacientes a quienes se les suministró Poractant alfa y be- 
ractant fueron los que presentaron bradicardia, reducción de la saturación de $\mathrm{O}_{2}$, hipotensión arterial o taquicardia en el $12,9 \%$ de los casos para cada uno. Singh N y cols., al comparar el Poractant alfa y beractant, no hallaron diferencias estadísticamente significativas en cuanto a incidencia de sepsis, enterocolitis necrotizante, ductus arteriosos permeable, retinopatía de la prematuridad severa (estados II-IV o que requiera terapia de fotocoagulación) y hemorragia intraventricular severa (grado III o IV) ${ }^{6}$. Baroutis G y cols., no hallaron diferencias estadísticamente significativas entre bovactant, Poractant alfa y beractant a dosis de $100 \mathrm{mg} / \mathrm{kg}$, para ductus arterioso permeable, síndromes de escape aéreo, retinopatía de la prematuridad, enterocolitis necrotizante y hemorragia intraventricular (grados $\geq$ II). Estos hallazgos son similares a los informados por Yalaz M y cols. ${ }^{9}$, quienes también compararon bovactant con beractant.

En este trabajo no se logró hallar diferencia estadísticamente significativa para mortalidad en las primeras $48 \mathrm{~h}$ de vida, a pesar de que estas fueron menos frecuentes en los neonatos que recibieron bovactant. La DBP y la combinación mortalidad y/o DBP, tampoco fue diferente entre los tres tipos de surfactante administrado. Sin embargo, esto pudo estar asociado al bajo número de pacientes en el estudio. Estos datos son similares a los informados en América Latina por Barría $\mathrm{M}$ y cols. ${ }^{24}$, para quienes la mortalidad global fue de $35 \%$, siendo mayor para los menores de 25 semanas $(86,7 \%)$, pero mayor a la informada en otro trabajo (16$18 \%)^{23}$. En un meta-análisis, se encontró una reducción estadísticamente significativa en la mortalidad favoreciendo al Poractant alfa en comparación con beractant a dosis de $200 \mathrm{mg} /$ $\mathrm{kg}$ y $100 \mathrm{mg} / \mathrm{kg}$ respectivamente (RR: 0,29; IC 95\%: 0,12-0,66; $\mathrm{p}<0,05)^{6}$, resultado no encontrado en nuestro trabajo. Singh N y cols. ${ }^{6}$, no hallaron diferencias en cuanto a incidencia de DBP cuando se suministraba Poractant alfa a $200 \mathrm{mg} / \mathrm{kg}$ y beractant a $100 \mathrm{mg} / \mathrm{kg}$ en su meta-análisis. Baroutis $\mathrm{G}$ y cols. ${ }^{8}$, en prematuros $\leq 32$ semanas de gestación con peso al nacer $\leq 2.000 \mathrm{~g}$ al nacer, compararon los resultados de bovactant, Poractant alfa y beractant a dosis de $100 \mathrm{mg} / \mathrm{kg}$, encontrando mor- talidad de 25,9\% para bovactant, 18,5\% para Poractant alfa y $23 \%$ para beractant, diferencia que no fue estadísticamente significativa. En este trabajo, tampoco hallaron diferencia estadísticamente significativa para DBP (bovactant $11,1 \%$, Poractant alfa $14,8 \%$ y beractant $15,4 \%$ ). Ramanathan $\mathrm{R}$ y cols. ${ }^{25}$, compararon la mortalidad en neonatos de 32 y menos semanas, con menos de $2.000 \mathrm{~g}$ de peso al nacer, tratados con Poractant alfa, calfactant y beractant, encontrando una significativa mayor mortalidad en los neonatos tratados con calfactant al compararse con Poractant alfa (OR: 1,496; IC 95\%: $1,014-2,209 ; p=0,043)$ y una tendencia a la mayor mortalidad en los pacientes tratados con beractant al compararase con Poractant alfa (OR: 1,370; IC 95\%: 0,996-1,885; $\mathrm{p}=0,053)$.

Hallamos que las tres preparaciones tuvieron beneficios clínicos similares con diferencias en algunos resultados, debido probablemente a varias razones:

1. Hay limitaciones metodológicas: La administración de los tres surfactantes no fue aleatoria ni cegada.

2. Debido al tiempo tan largo del estudio, algunos parámetros en la administración del surfactantes y cambios en las conductas ventilatorias y de oxigenación se han modificado, lo que puede verse reflejado en los diferentes resultados.

3. Teniendo en cuenta las recomendaciones actuales, los tres surfactantes se administraron a diferentes dosis, siendo mayor para Poractant alfa.

A pesar de las diferencias existentes en la composición, propiedades biofísicas y dosis de los tres surfactantes, se halló diferencia para algunas variables subrogadas, pero no se hallaron diferencias estadísticamente significativas en los resultados primarios de los neonatos y consideramos, como lo menciona Yudkin JS y cols. ${ }^{25}$, que la fiabilidad de las variables subrogadas para predecir con exactitud el resultado de una terapia es cuestionado y debemos prestar mucha atención a los resultados finales, como es en este caso, mortalidad, DBP, tiempo de oxigenoterapia, estancia hospitalaria y costo-efectividad de la terapia suministrada. 
Sin embargo, reconocemos que el tamaño de muestra es limitado para responder las preguntas sobre los resultados en cuanto a DBP y mortalidad.

Se hace necesario realizar un ensayo clínico, aleatorizado, randomizado y cegado, con tamaños de muestra mayores, que compare los resultados de la administración de los diferentes surfactantes, incluyendo una dosis mayor para el bovactant, la cual se pueda comparar de manera más justa con el Poractant alfa a $200 \mathrm{mg} / \mathrm{kg}$. Además se debe, en una futura investigación, incluir el tratamiento con presión positiva continua de la vía aérea (CPAP), tanto en prematuros menores de $1.500 \mathrm{~g}$, como en aquellos de más peso, tal como lo demostró Tapia JL y cols., quien informa que esta terapia asociada a la técnica INSURE (intubación, surfactante, extubación) resultó efectiva en el tratamiento de la EMH, con reducción de la necesidad de ventilación mecánica y requerimiento de surfactante, sin aumentar las complicaciones, la displasia broncopulmonar y la mortalidad $^{27}$.

\section{Referencias}

1.- Sinha S, Gupta S, Donn S: Immediate respiratory management of the preterm infant. Seminars in Fetal and Neonatal Medicine 2008; 13: 24-9.

2.- Soll RF: Prophylactic synthetic surfactant for preventing morbidity and mortality in preterm infants. Cochrane Database Syst Rev 2000; (2): CD001079.

3.- Soll R, Blanco F: Natural surfactant extract versus synthetic surfactant for neonatal respiratory distress syndrome. Cochrane Database Syst Rev 2001; (2): CD000144.

4.- Soll R: Synthetic surfactant for respiratory distress syndrome in preterm infants. Cochrane Database Syst Rev 2000; (2): CD001149.

5.- $\quad$ Soll R, Özek E: Multiple versus single doses of exogenous surfactant for the prevention or treatment of neonatal respiratory distress syndrome. Cochrane Database of Systematic Reviews 1999, Issue 2. Art. No.: CD000141. DOI: $10.1002 / 14651858 . C D 000141$.

6.- $\quad$ Singh N, Hawley K, Viswanathan K: Efficacy of Porcine Versus Bovine Surfactants for Preterm Newborns With Respiratory Distress Syndrome: Systematic Review and Meta-analysis. Pediatrics 2011; 128; e1587-e1595.

7.- Sweet DG, Carnielli V, Greisen $G$, et al: European
Consensus Guidelines on the Managenemt of Neonatal Respiratory Distress Syndrome in Preterm Infants-2010 Update. Neonatology 2010; 97: 402-17.

8.- Baroutis G, Kaleyias J, Liarou T, Papathoma E, Hatzistamatiou Z, Costalos C: Comparison of three treatment regimens of natural surfactant preparations in neonatal respiratory distress syndrome. Eur J Pediatr 2003; 162: 476-80

9.- Yalaz M, Arslanoglu S, Akisu M, Atik T, Ergun O, Kultursay $N$ : A comparison of efficacy between two natural exogenous surfactant preparations in premature infants with respiratory distress syndrome. Klin Padiatr 2004; 216: 230-5.

10.- Fujii A, Patel S, Allen R, Doros G, Guo C, Testa S: Poractant alfa and beractant treatment of very premature infants with respiratory distress syndrome. J Perinatol 2010; 30 (10): 665-70.

11.- Speer CP, Gefeller O, Groneck P, et al: Randomised clinical trial of two treatment regimens of natural surfactant preparations in neonatal respiratory distress syndrome. Arch Dis Child Fetal Neonatal Ed. 1995; 72 (1): F8-F13.

12.- Proquitté H, Dushe Th, Hammer H, Rüdiger M, Schmalisch $G$, Wauer RR: Observational study to compare the clinical efficacy of the natural surfactants Alveofact and Curosurf in the treatment of respiratory distress syndrome in premature infants. Respiratory Medicine 2007; 101: 169-76.

13.- Suresh GK, Soll RF: Overview of surfactant replacement trials. J Perinatol 2005; 25: S40-S44.

14.- Park J, Bae ChW, Choi YM: In vitro inhibition of Biophysical surface properties and change in ultrastructures of exogenous pulmonary surfactant by albumino $r$ fibrinogen. J Korean Med Sci 1998; 13: 123-30.

15.- Wang Z, Notter RH: Additivity of protein and nonprotein inhibitors of lung surfactant activity. Am J Respir Crit care Med 1998; 158: 28-35.

16.- Schmiedl A, Krug N, Hohlfeld JM: Influence of plasma and inflammatory proteins on the ultraestructure of exogenous surfactant. Journal of Electron Microscopy 2004; 53 (4): 407-16.

17.- Speer $C P$ : Chorioamniotitis, postnatal factors and proinflammatory response in the pathogenetic sequence of bronchopulmonary dysplasia. Neonatology 2009; 95 : 353-61.

18.- Dunn MS, Shennan AT, Possmayer F: Single-versus multiple-dose surfactant replacement therapy in neonates of 30 to 36 weeks' gestation with respiratory distress syndrome. Pediatrics 1990; 86 (4): 564-71.

19.- Speer CP, Robertson B, Curstedt T, et al: Randomized 
European multicenter trial of surfactant replacement therapy for severe neonatal respiratory distress syndrome: single versus multiple doses of Curosurf. Pediatrics 1992; 89 (1): 13-20.

20.- Soll RF: Multiple versus single dose natural surfactant extract for severe neonatal respiratory distress syndrome (Cochrane Review). In: The Cochrane Library. Issue 3. Oxford: Update Software; 2003.

21.- van Kaam AH, De Jaegere AP, Borensztajn D, Rimensberger PC: on behalf of the Neovent Study Group. Neonatology 2011; 100: 71-7.

22.- Moura C, Ferreira L, Agostini A, Tavares A: Timing of initial surfactant treatment in very low bitrh weight newborns. Einstein 2010; 8 (3 Pt 1): 320-4.

23.- Koch L, Frommhold D, Beedgen B, Ruef P, Poeschl $J$ : Prophylactic Administration of Surfactant in extremely Premature Infants. Critical Care Research and Practice 2010, Article ID 235894, 5 páginas, doi:10.1155/2010/235894

24.- Barría M, Pino P, Becerra C: Mortalidad en prematuros tratados con surfactante exógeno. Rev Chil Pediatr 2008; 79 (1): 36-44.

25.- Ramanathan R, Bhatia JJ, Sekar K, Ernst FR: Mortality in preterm infants with respiratory distress syndrome treated with Poractant alfa, calfactant or beractant: a retrospective study. J Perinatol 2013; 33: 119-25.

26.- Yudkin JS, Lipska KJ, Montori VM: The idolatry of the surrogate. BMJ 2011; 343: d7995. doi: 10.1136/bmj. d7995.

27.- Tapia JL, Urzúa S, Bancalari A, et al: Randomized Trial of Early Bubble Continuous Positive Airway Pressure for Very Low Birth Weight Infants. J Pediatr 2012; 161: 75-80. 